\title{
The effect of viscous loading on brain ependymal cilia
}

Christopher Liam O’Callaghan ", Kulvinder Sikand, Andrew Rutman and Robert Anthony Hirst

Department of Infection, Immunity and Inflammation

University of Leicester

Robert Kilpatrick Clinical Sciences Building

Leicester Royal Infirmary

Leicester LE2 7LX

UK

* Corresponding author

Professor Chris O’Callaghan

Department of Infection, Immunity and Inflammation

University of Leicester

Robert Kilpatrick Clinical Sciences Building.

PO Box 65, Leicester Royal Infirmary

Leicester LE2 7LX

UK

Tel: $\quad 01162523268$

Fax: $\quad 01162523282$

E-mail: ajb64@le.ac.uk

Key Words: Viscous, Brain ependymal cilia, Ciliary beat frequency, Ciliary beat amplitude

Length: 15 pages including 3 figures.

Word count of manuscript: 3,890

Abbreviations: CBF: ciliary beat frequency; CSF: cerebrospinal fluid; PCD: primary ciliary dyskinesia 


\begin{abstract}
Ependymal cilia line the ventricular system moving cerebral spinal fluid close to the brain surface. They may be exposed to fluid of increasing viscosity in certain pathological conditions such as bacterial meningitis. Our aim was to determine the effect of increasing viscosity on ciliary function.
\end{abstract}

Ciliated ependyma was exposed to solutions of different viscosities (1-60 cP) and ciliary function assessed by highspeed digital imaging.

The mean (SD) ciliary beat frequency (CBF), measured after 30 minutes incubation in Medium 199 at $37^{\circ} \mathrm{C}$, was 34.9 (2.9) Hz. Increased viscous loading was followed by a rapid decrease in ciliary beat frequency compared to baseline readings $(\mathrm{p}<0.001)$. After 15 minutes of exposure to the increased viscous load, CBF reached a new stable level while the viscous load was maintained. Compared to baseline measurements of $\mathrm{CBF}$, viscous loading of $3.7 \mathrm{cP}$ caused a $16 \%$, $10.4 \mathrm{cP}$ at $34 \%$ and $24 \mathrm{cP}$ a $70 \%$ decrease in beat frequency. Further viscous loading at levels up to $60 \mathrm{cP}$ resulted in no further reduction of ependymal CBF. Solutions of $24 \mathrm{cP}$ and $40 \mathrm{cP}$ had no effect on ciliary amplitude. An increase in viscosity to $60 \mathrm{cP}$ caused a significant $(30 \%: \mathrm{p}=0.001)$ decrease in the ciliary beat amplitude. 


\section{Introduction}

The physiological roles of ependymal cilia are still poorly understood. Beating ependymal cilia move CSF, in close proximity to the cerebral ventricles and aqueducts they line, in a predictable direction $[4,22]$. Recent work suggests that the movement of CSF by ependymal cilia is required for the establishment of a concentration gradient of CSF guidance molecules that direct the migration of neuroblasts to various brain regions[21]. Ependymal cilia are also thought to play a role in host defence keeping the surface of the brain clear of debris. It is of interest that abnormal ciliary function has been associated with the development of ventricular dilatation and hydrocephalus in a number of studies $[2,8,12,16]$. We have recently shown that intracerebroventricular antisense knockdown of $\mathrm{G} \alpha_{\mathrm{i} 2}$ results in ciliary stasis and ventricular dilatation in rats [16]. An inhibitor of ciliary movement, metavanadate, has also been shown to induce hydrocephalus in rats [17]. Hydrocephalus is also seen in patients and especially small animals with primary ciliary dyskinesia (PCD) [12,13]. PCD is an inherited condition where all of the body's normally motile cilia are either stationary or move in a dyskinetic way that makes them inefficient in propelling fluid or mucus. Administration of virus or bacteria to the CSF, in animal models of meningitis, has been associated with ependymal damage and hydrocephalus $[10,11]$. Whilst bacteria and their toxins $[6,7,15]$ have been shown to adversely affect ciliary function other factors including the inflammatory response may have contribute to the toxicity seen. It is known that CSF protein can alter the viscocity of the CSF [3] however the effect of meningitis induced increases in CSF protein on ependymal ciliary function has not been studied.

The aim of this study was to determine the effects of viscosity on ependymal ciliary function as measured by changes in ciliary beat frequency and beat amplitude. 


\section{Materials and methods}

\section{Tissue preparation:}

The brains of Wistar rats (9-15 days of age) were dissected following sacrifice by cervical dislocation. Coronal brain slices from the floor of the 4th ventricle of Wistar rats (10-15 days of age) were prepared immediately after sacrifice and mounted in a well containing $4 \mathrm{ml}$ of Medium 199 with Earl's salts (pH7.4: plus penicillin 50u/ml and streptomycin $50 \mu \mathrm{g} / \mathrm{ml})$. This preparation resulted in a reproducible side on profile of aligned ependymal cells and cilia. Samples were enclosed in a purpose designed environmental chamber which maintained the solution at $37^{\circ} \mathrm{C}$, and the surrounding air at humidity at $80 \%$ to minimise evaporation. Ciliary movement was observed using a x50 water immersion lens using Kohler illumination on a diaphot inverted microscope (Nikon, UK).

\section{Measurement of ciliary beat frequency:}

Beating ciliated edges were recorded by a high-speed video camera (Kodak EktaPro Motion Analyser, Model 1012) at a rate of 400 frames per second. The camera allowed video sequences to be down loaded at reduced frame rates, allowing ciliary beat frequency to be determined directly by timing a given number of individual ciliary beat cycles. At each time point of the study ciliary beat frequency was measured at 4 different areas along each ependymal strip. Only intact, undisrupted, ciliated strips in excess of $100 \mu \mathrm{m}$ were studied.

\section{Viscosity measurement:}

Viscosity was determined in accordance with the British Standards guidelines (1977) using an Ubbelohde viscometer.

The kinematic viscosity was calculated from the measured flow time and the calibration factor of the viscometer. The density was measured at the same temperature as the determination of kinematic viscosity allowing the product of the two, the dynamic viscosity at that temperature, to be calculated.

Kinematic viscosity $(v) . \quad v=\mathrm{C} \mathrm{t}$

where $\mathrm{t}$ is the mean flow time (in seconds); $\mathrm{C}$ is the factor for the viscometer, given in its calibration certificate.

The result is expressed in $\mathrm{mm}^{2} / \mathrm{s}$. The SI unit of kinematic viscosity is the metre squared per second $\left(\mathrm{m}^{2} / \mathrm{s}\right)$

Dynamic viscosity $(n) . \quad \mathrm{n}=V \mathrm{p}$ 
where $v$ is the kinematic viscosity (in $\mathrm{mm}^{2} / \mathrm{s}$ ), $\mathrm{p}$ is the density measured at the same temperature (in $\mathrm{g} / \mathrm{cm}^{3}$ )

The SI unit of dynamic viscosity is the Pascal second ( $\mathrm{Pa} \mathrm{s})$. The millipascal second ( $\mathrm{mPa} s)$ is equal to the centipoise (cP). Viscosity in this study are reported in units of $\mathrm{cP}$ to allow easier reference to previous investigations into the effect of viscosity on ciliary function.

Repeatability; Duplicate results by the operator using the same viscometer showed that their difference was less than $0.35 \%$ of their mean.

The type of direct-flow viscometers we used were Ubbelohde, with calibration constants of 0.004988, 0.02914 and $0.3172 \mathrm{~mm}^{2} / \mathrm{s}^{2}$

\section{Effect of viscous loading on ciliary beat frequency:}

Ependymal strips were incubated at $37^{\circ} \mathrm{C}$ for 15 minutes prior to initial readings. A second reading was made 15 minutes later. The Medium 199 bathing the ependyma was then exchanged with one of the experimental solutions. Those conducting the experiment were blinded to the nature of the solution. Solutions of the following viscosities were used; $3.7 \mathrm{cP}, 10.4 \mathrm{cP}, 25.3 \mathrm{cP}, 40 \mathrm{cP}$ and $60 \mathrm{cP}$. A solution of Medium 199 (1cP) was used as a control. All solutions were preheated to $37^{\circ} \mathrm{C}$ prior to exchange. Readings of ciliary beat frequency were taken at 3, 15, 30, 45 and 60 minutes after exchange.

The higher concentrations of dextran (Sigma, Poole, UK) resulted in a low measured sodium (95 mmol/1) and a high osmolality $(375 \mathrm{mmol} / \mathrm{kg})$. To determine whether a solution of low viscosity $(1 \mathrm{cP})$, high osmolality and low sodium (95mmol/1), also caused ciliary slowing the following experiment was conducted. A solution of artificial CSF (in mmol/l; NaCl 125 mmol/l, KCI 2.5, mmol/l, Glucose 10 mmol/l, Hepes 10 mmol/l, $\mathrm{MgCl}_{2} 1 \mathrm{mmol} / \mathrm{l}, \mathrm{CaCl}_{2} 2 \mathrm{mmol} / \mathrm{l}$ ) adjusted to a $\mathrm{pH} 7.3$ was made. The amount of sucrose required to increase the osmolality up to 375 , and to produce a solution with only $90 \mathrm{mmol} / \mathrm{l}$ of $\mathrm{Na}$ was determined and the dynamic viscosity measured.

\section{Effect of viscous loading on the range of ciliary motion:}

The effect of viscous load on the distance travelled by the tip of a single ependymal cilium during its power stroke was determined. Brain slices were stored in Medium 199 with Earl's salts (pH7.4: plus penicillin 50u/ml and streptomycin $50 \mu \mathrm{g} / \mathrm{ml}$ ), at $37^{\circ} \mathrm{C}$ for 30 minutes, and ciliary motion recorded at a frame rate of 400 per second. The bathing solution was then exchanged for a preheated $\left(37^{\circ} \mathrm{C}\right)$ solution of either $24 \mathrm{cP}(\mathrm{n}=7), 40 \mathrm{cP}(\mathrm{n}=6)$, or $60 \mathrm{cP}(\mathrm{n}=8)$. Ciliary motion was recorded, again, after twenty minutes. The position of the cilium (magnified 1500 times) was stored at intervals of 
2.5 milliseconds. The distance travelled by the tip of the cilium was measured during its forward stroke. This was divided by the ciliary length. This was to offset any differences in measurement due to the increased refractive index of the more viscous solutions. This index was used to compare initial movement with that following fluid exchange with results expressed as a percentage of the cilium's movement in Medium 199.

\section{Electron Microscopy:}

The brain slices were fixed in Sorensens phosphate buffered ( $\mathrm{pH} 7.4)$ gluteraldehyde (4\%w/v). After post-fixation in osmium tetroxide $(1 \% \mathrm{w} / \mathrm{v})$, samples were dehydrated through graded ethanols and immersed in hexamethyldisilazane (HMDS). The HMDS evaporated leaving dry tissue with no phase boundary damage (damage caused to the tissue by pressure differences of the air/liquid interface).

\section{Statistical analysis:}

A split unit analysis of variance was performed with two factors, viscosity and time. The effect of viscosity was assessed relative to the between slice variability, while the effects of time and the interaction between viscosity and time were assessed relative to the within slice variability. To determine the effect of exchanging the control medium with an artificial CSF or with a low sodium CSF, the means of the duplicate values were analysed. Paired t tests were performed to compare artificial CSF (aCSF) with control and low sodium CSF with control. A two-sample t test was used to compare the mean change from control for the two media.

To determine the effect of changing viscosity on beat amplitude a one way analysis of variance was used to compare the three viscosities $(24,40$, and $60 \mathrm{cP})$ studied. The Bonferroni procedure was used to compare every pair of means. For each viscosity a one sample t-test was used to compare results to readings obtained in Medium 199. 


\section{Results}

The mean (SD) baseline ciliary beat frequency measured after 30 minutes incubation in Medium 199 solution at $37^{\circ} \mathrm{C}$ was 34.9(2.9) Hz. Increased viscous loading was followed by a rapid decrease in frequency of ependymal cilia. After 15 minutes of exposure to the increased viscous load the beat frequency reached a new equilibrium value that remained fairly stable while the viscous load was maintained.

The beat frequency decreased to $84 \%$ of base line readings with the addition of a solution with a viscosity of $3.7 \mathrm{cP}$ to $66 \%$ at $10.4 \mathrm{cP}$ and $30 \%$ at $24 \mathrm{cP}$. Further viscous loading at levels up to $60 \mathrm{cP}$ resulted in no further suppression of ependymal ciliary frequency, however the ciliary beat amplitude was decreased. When the analysis was restricted to viscosities of $24 \mathrm{cP}$ or more there was no significant interaction between viscosity and time $(\mathrm{P}=0.29)$ and no significant effect of viscosity $(\mathrm{P}=0.47)$. The time course of the effect of viscous loading on the frequency of ependymal cilia is shown in Figure 1 A-F. The dose finding graph for the inhibition of ciliary beat frequency at 90 minutes (Figure 2A), indicated an approximate $50 \%$ inhibitory viscosity of $13 \mathrm{cP}$.

The effect of viscous load on ciliary beat amplitude after equilibration at $24 \mathrm{cp}, 40 \mathrm{cP}$ and $60 \mathrm{cP}$ compared to base line readings are shown in figure $2 \mathrm{~B}$. The analysis of variance used to compare the effect of the 3 viscosities $(24,40$, and $60 \mathrm{cP})$ on ciliary amplitude showed highly significant differences between the three means $(\mathrm{p}<0.001)$. The highest viscosity was significantly different from either of the other two viscosities. A one sample t test comparing the mean $\%$ amplitude with $100 \%$ was only significant for the $60 \mathrm{cP}$ solution.

There was no significant difference in ciliary beat frequency between the artificial CSF and control or between low sodium CSF and control (data not shown). Exchange of Medium 199 with artificial CSF or artificial CSF with a high osmolality (388mosmol/1) and low sodium concentration $(91 \mathrm{mmol} / \mathrm{l})$ resulted in a $9.8 \%$ and $7 \%$ increase in ciliary beat frequency respectively.

Scanning electron microscopy revealed even brain sections through the floor of the $4^{\text {th }}$ ventricle. Ciliated ependymal cells were present over the entire floor of the ventricle (Figure $3 \mathrm{~A}$ ). The ependymal cilia were approximately $8 \mu \mathrm{m}$ in length and each cell had about 40cilia (Figure 3B). The alignment of the cilia was predictive of CSF directional flow (Figure $3 \mathrm{~A}$ and $\mathrm{B})$. 


\section{Discussion}

We have shown that increasing the viscosity of fluid surrounding brain ependymal cilia results in a rapid decrease in ciliary beat frequency. Once this rapid decrease in beat frequency occurred cilia beat at their new, lower, frequency for the remainder of the study period. Viscous loading of $3.7 \mathrm{cP}, 10.4 \mathrm{cP}$ and $24 \mathrm{cP}$ caused a progressive decrease in ciliary beat frequency. At viscosities up to $40 \mathrm{cP}$ the distance moved by the cilia tip during its active stroke, the ciliary amplitude, did not change. However, while further viscous loading of up to 60cP did not depress beat frequency further, this viscosity did significantly decrease the ciliary beat amplitude.

Other than the observed effects on ciliary beat frequency and beat amplitude, there was very little visible effect of the viscous loading on the coordination of the ciliary beat cycle.

Our results are not dissimilar to those of Machemer, who showed that in the water propelling cilia of Paramecium, as viscosity is raised the polarization of the ciliary cycle in time and space is reduced, and the frequency of peristomal cilia decreases with rising viscosity [14]. Machemer found the percentage decrease in beat frequency with viscous loading was similar to the decrease in frequency seen in this study when similar viscous loads were applied to ependymal cilia. It must be noted however, that our data are not directly comparable with Machemar because ependymal cilia have a different cellular function to the cilia of the paramecium.

Although water-propelling cilia do appear to be able to adapt to increasing viscosity they do not appear to be as well adapted as mucous propelling respiratory cilia [9]. Johnson and colleagues [9] found that mucous propelling cilia of the respiratory tract from confluent clusters of cells or single cells showed autoregulatory properties when exposed to an increased viscous load. Despite the use of viscous loads, that were considerably higher $(1-150 \mathrm{cP})$ than those used in our experiments, the beat amplitude and frequency exhibited only slight variations over viscosities ranging from $12-150 \mathrm{cP}$. Johnson and colleagues found that when they increased the viscous load beyond 180cP, ciliary activity became unpredictable [9]. Cilia on some cells stopped beating whilst others were observed to develop a fibrillatory beat pattern of high frequency and short amplitude. Andrade and colleagues also found ciliary beat frequency decreased with increasing viscosities up to $30 \mathrm{cP}$ but there was no further decrease in frequency at viscosities up to 210cP [1]. This ability to auto regulate activity is not secondary to an external nerve mediated reflex as cultured monolayers are devoid of nerve terminals. It is not immediately clear why ependymal cilia did not tolerate viscous loading as well as respiratory cilia. There are, however, significant differences between ependymal and respiratory cilia. Ependymal cilia 
are not normally subject to varying viscous loads and the flow of the cerebral spinal fluid is constant. There are only 40 cilia per ependymal cell compared to 200 cilia on ciliated respiratory cells and the basal beat frequency of ependymal cells is approximately twice that of respiratory cilia. Ependymal cilia are also longer than respiratory cilia. We are confident of our observations as the use of modern high speed digital imaging gave excellent resolution allowing us to determine beat frequency by direct observation and to assess beat pattern. This is a major advantage over the systems used in the study by Johnson and Andrade that relied on measurement of ciliary beat frequency using indirect methods $[1,9]$.

It is unlikely, in our study, that the ciliary activity decreased the apparent viscosity of the Medium 199-dextran solutions as such solutions have been shown to behave as Newtonian fluids when exposed to shear rates much higher than those produced by ciliary activity. The possibility of the lower sodium and higher osmolality of the high dextran solutions being responsible for the decrease in ciliary beat frequency is also unlikely as a solution of similar sodium concentration and osmolality but of low viscosity did not affect ciliary beat frequency.

The ability of cilia to respond to increasing viscous loading may be due to cellular reactions to changes in mechanical load or be due to inbuilt properties of the ciliary axoneme. Ciliated cells are mechanosensitive. Stimulation of the apical surface of a single ciliated cell with a glass microprobe elevates the ciliary beat frequency of the stimulated and adjacent cells $[19,20]$. The increase in beat frequency of each cell occurs after a lag-phase and is proportional to the distance from the stimulated cell. Stimulation of a non-ciliated cell, adjacent to ciliated cells, also increased their beat frequency. A mechanical stimulus to either a ciliated or non-ciliated cell induces an immediate increase in intra cellular $\mathrm{Ca}^{2+}$ at the contact point which spreads throughout the cell. After approximately half a second, a wave of increasing intracellular $\mathrm{Ca}^{2+}$ occurs in adjacent cells travelling across up to 7 cells. The rise in intracellular $\mathrm{Ca}^{2+}$ always preceded an increase in ciliary beat frequency [18]. The axoneme may also play a role as demembranated-ATP-reactivated axonemes of newt lung ciliated cells, under conditions in which calcium concentrations are kept constant, appear to autoregulate beat frequency under increased viscous loads [5]. Insight into the mechanism of autoregulation of ciliary beat frequency following exposure to changes in viscosity has been provided in a recent study by Andrade and colleagues [1]. They found that changes in mucous viscosity activates transient receptive potential vanilloid 4 (TRPV4) like channel that elevates intracellular $\mathrm{Ca}_{2+}$. They also found channel opening requires the activity of Phosphalipase $\mathrm{A}_{2}$ $\left(\mathrm{PLA}_{2}\right)$ and occurs at high viscous loads allowing the cilia to adapt to a wide range of viscosities. 
The importance of our findings in relation to mechanisms that occur in meningitis can only be inferred. We have successfully collected CSF from an adult rat with meningitis, but due to the very low volumes collected $(0.2 \mathrm{ml}$ maximum) we were unable to use it in our studies. In summary here we show that increasing viscosity of the CSF or viscous material found in the ventricular system will adversely affect ependymal ciliary function. 


\section{Figure Legends:}

\section{Figures 1:}

Time course of the effect of viscous loading on the ciliary beat frequency of ependymal cells. After a 30-minute control period in Medium 199, the ciliated ependymal cells were exposed to solutions of Medium 199 and dextran with different viscosities (1-60cP; A-F). Each line on a graph represents an independent experiment and each point on the graph is the mean reading of four independent cilia from the brain slice.

\section{Figure 2:}

The effect of viscous load on the CBF inhibition at 90 minutes (A) and the distance travelled by the tip of an ependymal cilia during its power stroke (B). For the amplitude measurements (B), each ciliated sample is the relative (\%) change of distance travelled by the cilia tip is referenced to its own control measured at the end of a 30 minute incubation period in Medium $199(1 \mathrm{cP})$.

Figure 3: Scanning electron micrograph of a representative vibrotome section through the floor of the $4^{\text {th }}$ ventricle (low power, A) and (high power, B). The geometry of the ciliated ependymal cells, the direction of CSF flow (red arrow, B) and the length of the cilia $(\sim 8 \mu \mathrm{m})$ can be seen. The blue scale bar $=10 \mu \mathrm{m}$. 


\section{References}

[1] Y.N. Andrade, J. Fernandes, E. Vazquez, J.M. Fernandez-Fernandez, M. Arniges, T.M. Sanchez, M. Villalon and M.A. Valverde, TRPV4 channel is involved in the coupling of fluid viscosity changes to epithelial ciliary activity, J Cell Biol 168 (2005) 869-874.

[2] B. Banizs, M.M. Pike, C.L. Millican, W.B. Ferguson, P. Komlosi, J. Sheetz, P.D. Bell, E.M. Schwiebert and B.K. Yoder, Dysfunctional cilia lead to altered ependyma and choroid plexus function, and result in the formation of hydrocephalus, Development 132 (2005) 5329-5339.

[3] H.L. Brydon, R. Hayward, W. Harkness and R. Bayston, Physical properties of cerebrospinal fluid of relevance to shunt function. 1: The effect of protein upon CSF viscosity, Br J Neurosurg 9 (1995) 639644.

[4] R.S. Cathcart, 3rd and W.C. Worthington, Jr., Ciliary Movement in the Rat Cerebral Ventricles: Clearing Action and Directions of Currents, J Neuropathol Exp Neurol 23 (1964) 609-618.

[5] R. Hard, K. Blaustein and P. Verdugo, The effect of viscous load on beat frequency of newt lung cilia., J Cell Biol 101 (1985) 269a.

[6] R.A. Hirst, B. Gosai, A. Rutman, P.W. Andrew and C. O'Callaghan, Streptococcus pneumoniae damages the ciliated ependyma of the brain during meningitis, Infect Immun 71 (2003) 6095-6100.

[7] R.A. Hirst, B.J. Mohammed, T.J. Mitchell, P.W. Andrew and C. O'Callaghan, Streptococcus pneumoniae-induced inhibition of rat ependymal cilia is attenuated by antipneumolysin antibody, Infect Immun 72 (2004) 6694-6698.

[8] I. Ibanez-Tallon, A. Pagenstecher, M. Fliegauf, H. Olbrich, A. Kispert, U.P. Ketelsen, A. North, N. Heintz and H. Omran, Dysfunction of axonemal dynein heavy chain Mdnah5 inhibits ependymal flow and reveals a novel mechanism for hydrocephalus formation, Hum Mol Genet 13 (2004) 2133-2141.

[9] N.T. Johnson, M. Villalon, F.H. Royce, R. Hard and P. Verdugo, Autoregulation of beat frequency in respiratory ciliated cells. Demonstration by viscous loading, Am Rev Respir Dis 144 (1991) 1091-1094.

[10] R.T. Johnson and K.P. Johnson, Hydrocephalus following viral infection: the pathology of aqueductal stenosis developing after experimental mumps virus infection, J Neuropathol Exp Neurol 27 (1968) 591 606.

[11] D.F. Kohn, B.E. Kirk and S.M. Chou, Mycoplasma-induced hydrocephalus in rats and hamsters, Infect Immun 16 (1977) 680-689. 
[12] M. Koto, J. Adachi and A. Shimizu, A new mutation of primary ciliary dyskinesia (PCD) with visceral inversion (Kartagener's Syndrome) and hydrocephalus., Rat News Lett 18 (1987) 14-15.

[13] M. Koto, M. Miwa, A. Shimizu, K. Tsuji, M. Okamoto and J. Adachi, Inherited hydrocephalus in Csk: Wistar-Imamichi rats; Hyd strain: a new disease model for hydrocephalus, Jikken Dobutsu 36 (1987) 157-162.

[14] H. Machemer, Ciliary activity and the origin of metachrony in Paramecium: effects of increased viscosity, J Exp Biol 57 (1972) 239-259.

[15] B.J. Mohammed, T.J. Mitchell, P.W. Andrew, R.A. Hirst and C. O'Callaghan, The effect of the pneumococcal toxin, pneumolysin on brain ependymal cilia, Microb Pathog 27 (1999) 303-309.

[16] K.S. Monkkonen, J.M. Hakumaki, R.A. Hirst, R.A. Miettinen, C. O'Callaghan, P.T. Mannisto and J.T. Laitinen, Intracerebroventricular antisense knockdown of $\mathbf{G}$ alpha $\mathrm{i} 2$ results in ciliary stasis and ventricular dilatation in the rat, BMC Neurosci 8 (2007) 26.

[17] Y. Nakamura and K. Sato, Role of disturbance of ependymal ciliary movement in development of hydrocephalus in rats, Childs Nerv Syst 9 (1993) 65-71.

[18] M.J. Sanderson, A.C. Charles and E.R. Dirksen, Mechanical stimulation and intercellular communication increases intracellular $\mathrm{Ca}^{2+}$ in epithelial cells, Cell Regul 1 (1990) 585-596.

[19] M.J. Sanderson, I. Chow and E.R. Dirksen, Intercellular communication between ciliated cells in culture, Am J Physiol 254 (1988) C63-74.

[20] M.J. Sanderson and E.R. Dirksen, Mechanosensitivity of cultured ciliated cells from the mammalian respiratory tract: implications for the regulation of mucociliary transport, Proc Natl Acad Sci U S A 83 (1986) 7302-7306.

[21] K. Sawamoto, H. Wichterle, O. Gonzalez-Perez, J.A. Cholfin, M. Yamada, N. Spassky, N.S. Murcia, J.M. Garcia-Verdugo, O. Marin, J.L. Rubenstein, M. Tessier-Lavigne, H. Okano and A. Alvarez-Buylla, New neurons follow the flow of cerebrospinal fluid in the adult brain, Science 311 (2006) 629-632.

[22] T. Yamadori and K. Nara, The directions of ciliary beat on the wall of the lateral ventricle and the currents of the cerebrospinal fluid in the brain ventricles, Scan Electron Microsc (1979) 335-340. 

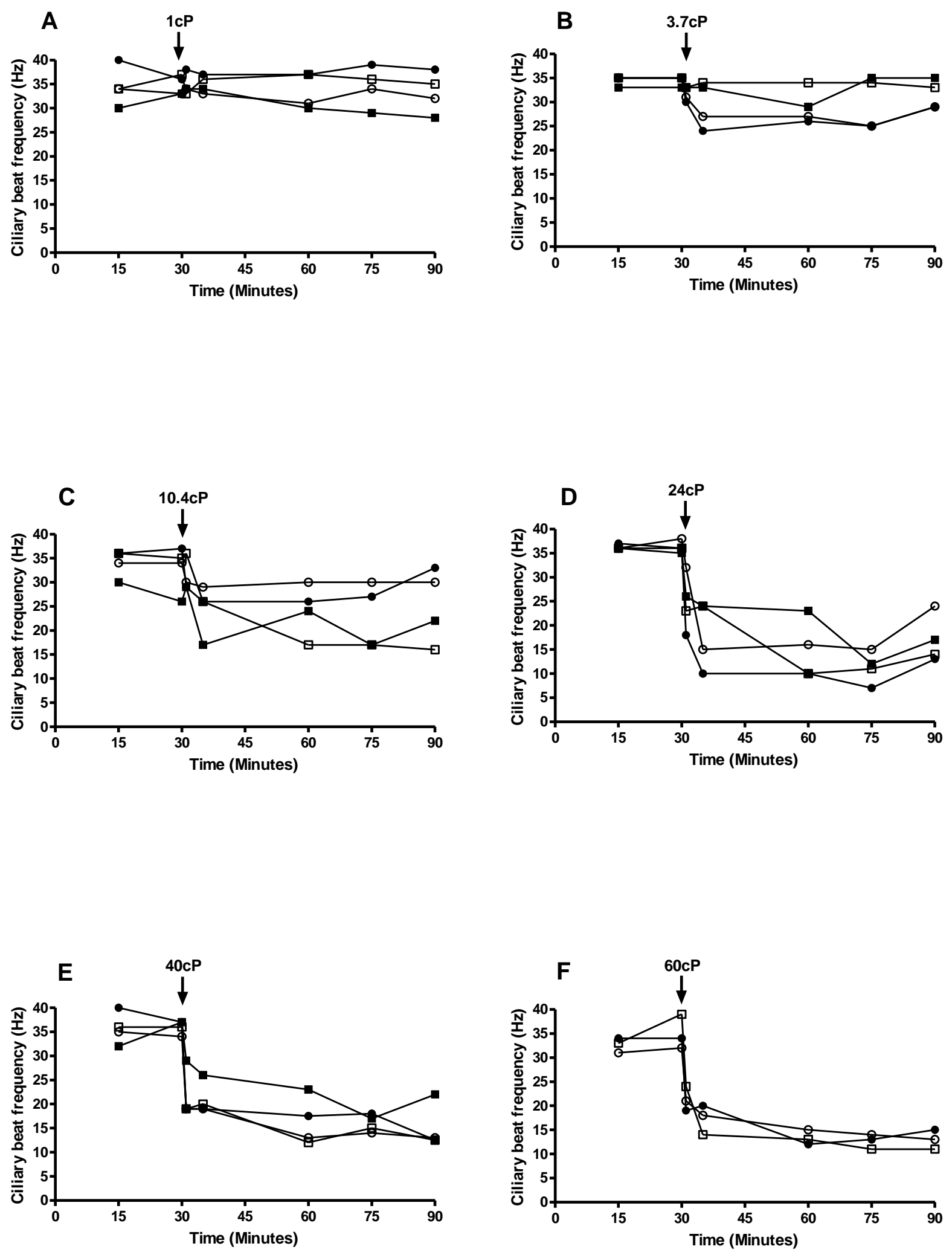

Figure 1 

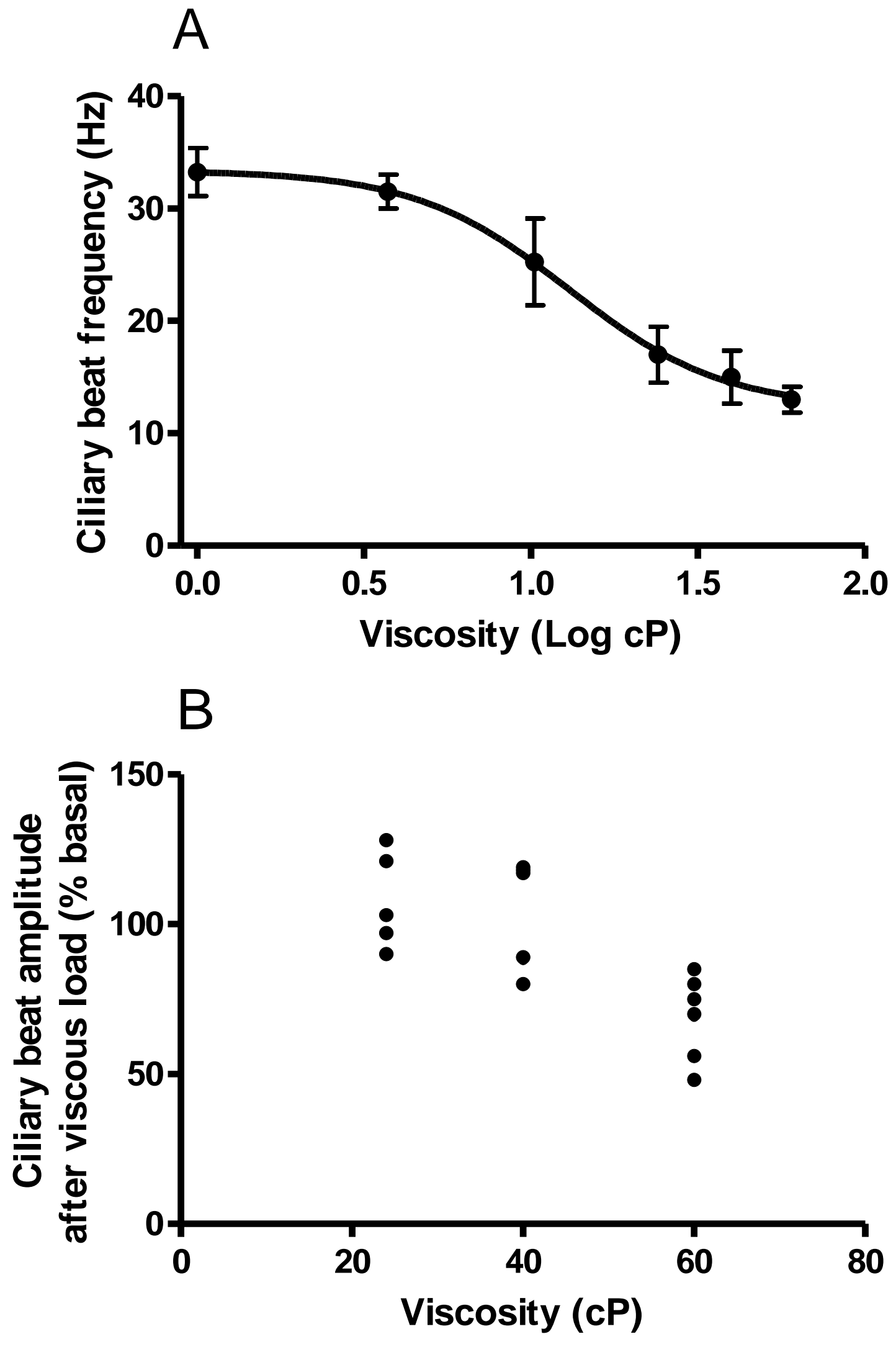

Figure 2 
Figure 3A

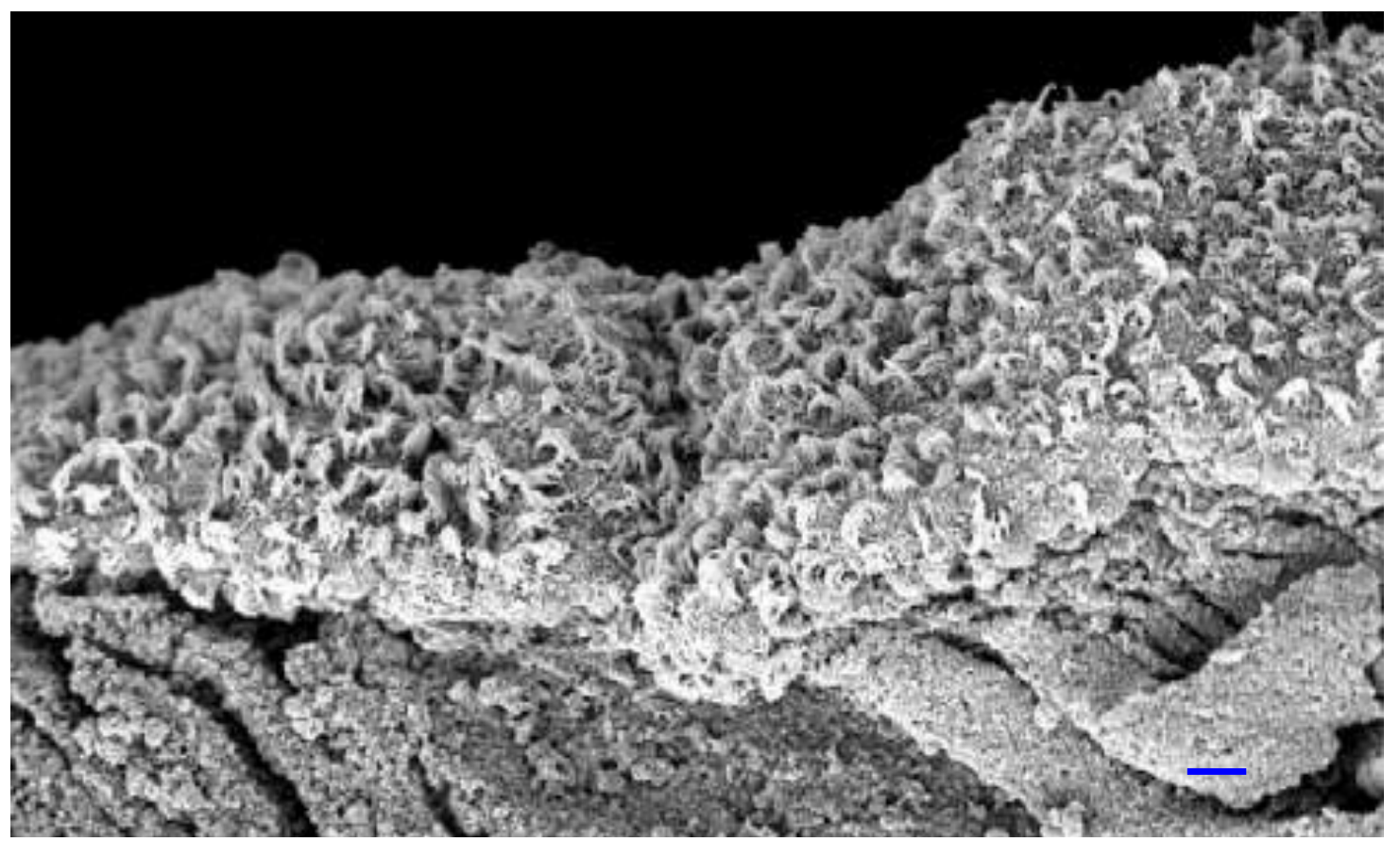

Figure 3B

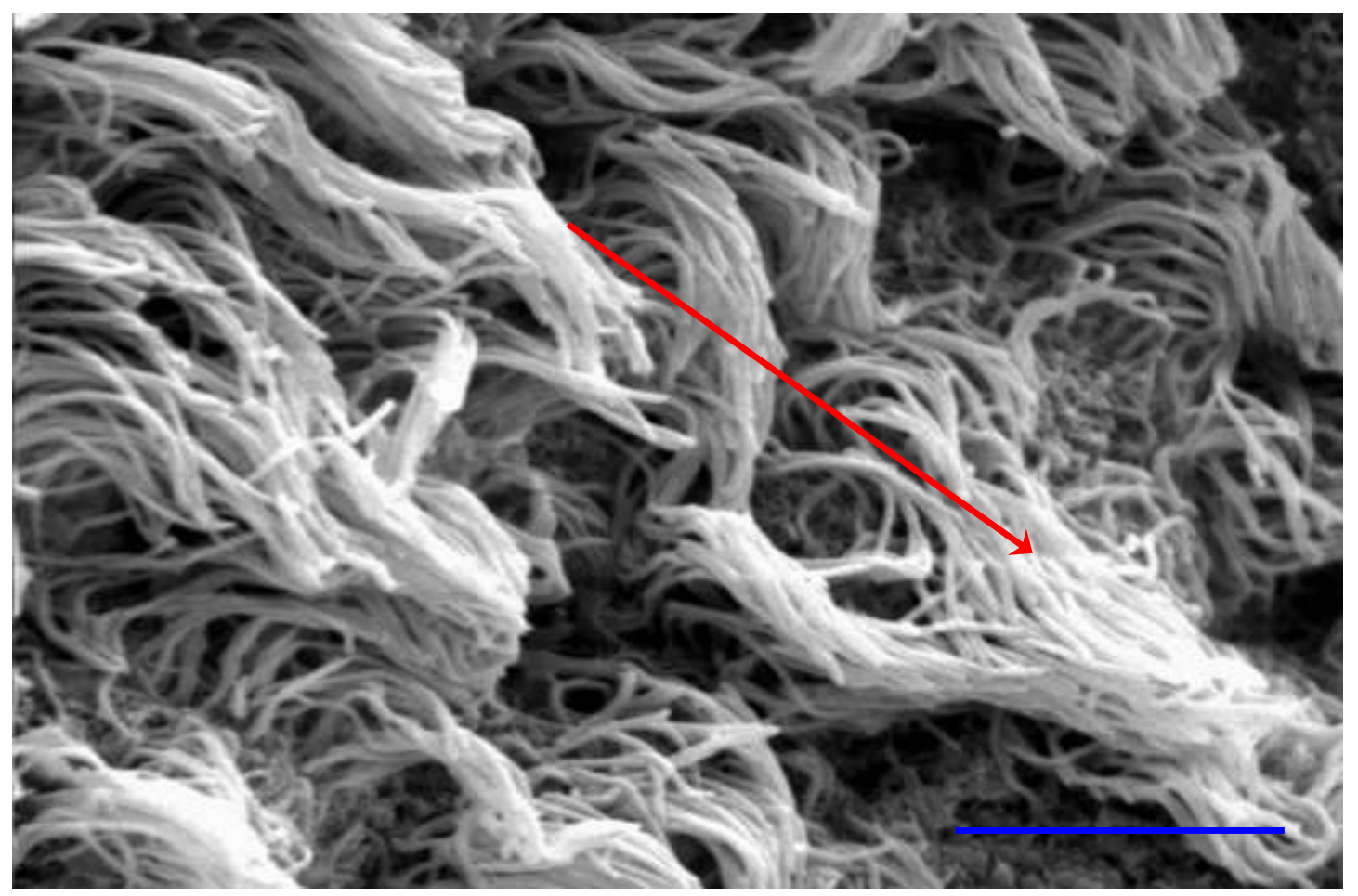

